\title{
A DATA ADAPTIVE COMPRESSED SENSING APPROACH TO POLARIMETRIC SAR TOMOGRAPHY
}

\author{
E. Aguilera, M. Nannini, and A. Reigber \\ Microwaves and Radar Institute, German Aerospace Center (DLR) \\ 82234 Wessling, Germany \\ Tel.: +49 - 8153281820, E-mail: Esteban.Aguilera@dlr.de
}

\begin{abstract}
Super-resolution imaging via compressed sensing (CS) based spectral estimators has been recently introduced to synthetic aperture radar (SAR) tomography. In the case of partial scatterers, the mainstream has so far been twofold, in that the tomographic reconstruction is conducted by either working directly with multiple looks and/or polarimetric channels or by exploiting the corresponding single-channel second order statistics. In this paper, we unify these two methodologies in the context of covariance fitting. In essence, we exploit the fact that both vertical structures as well as the unknown polarimetric signatures can be approximated in a low dimensional subspace. For this purpose, we make use of a wavelet basis in order to sparsely represent vertical structures. Additionally, we synthesize a data adaptive orthonormal basis that spans the space of polarimetric signatures. Finally, we validate this approach by using fully polarimetric L-band data acquired by the E-SAR sensor of the German Aerospace Center (DLR).
\end{abstract}

Index Terms - Compressed sensing (CS), distributed CS (DCS), polarimetry, synthetic aperture radar (SAR) tomography, wavelets, kronecker basis.

\section{INTRODUCTION}

Multibaseline polarimetric measurements allow us to resolve a vertical structure via well-established synthetic aperture radar (SAR) imaging principles. Also, they provide an additional dimension to further describe the response of illuminated objects, which, in the case of partial scatterers, is commonly captured in the form of a polarimetric covariance matrix. However, the achievable resolution of conventional estimators is highly dependent on the extension of the elevation aperture. Moreover, the sampling rate dictated by the well-known Nyquist frequency imposes an additional requirement, namely dense, regular sampling [1].

Recently, alternative sparsity driven non-linear reconstruction algorithms have been put forward in order to attain low sidelobe and ambiguity levels with a reduced number of irregular passes. In particular, the authors in [2] extended the work in $[3,4]$ and proposed a compressed sensing (CS) based joint reconstruction technique that takes advantage of possible intersignal structural correlations between neighboring azimuth-range pixels as well as between polarimetric channels. Also, a single-channel covariance fitting methodology was introduced in [5], that employs sparse representations of the vertical backscattered power in the wavelet domain. In this paper, we combine all these lines of research and introduce the concept of polarimetric signature awareness, thus not only exploiting structural correlations, but also taking advantage of the fact that polarimetric signatures, as characterized by the so-called coherency/covariance matrix (from now on referred to as polarimetric covariance matrix) [6], can be approximated in a low dimensional subspace [7]. For this purpose, we form a data adaptive orthonormal basis that spans the space of polarimetric signatures.

The remainder of the paper is organized as follows. In Section 2, we formulate the inverse problem from a multibaseline polarimetric covariance matrix perspective in such a way that we are able to decouple the scattering mechanisms' structures from their polarimetric signatures. Subsequently, Section 3 revisits the concept of joint sparse reconstruction. Section 4 casts the covariance fitting problem as an instance thereof and reformulates it so as to be able to restrict the polarimetric signatures to lie in a low dimensional subspace. In Section 5, we present results obtained using fully polarimetric L-band data acquired by one of the airborne sensors of the German Aerospace Center (DLR), namely E-SAR. Lastly, Section 6 concludes the paper.

\section{PROBLEM FORMULATION}

Let $K_{i, j} \in \mathbb{C}^{m \times m}$ be the multibaseline covariance matrix resulting from $m$ parallel passes $[1,8]$ and two polarimetric channels $i$ and $j$ at a specific azimuth-range position; with $1 \leq i, j \leq 3$. For example, $i$ and $j$ could denote the $h h$ and $h v$ channels, respectively. Then, we can construct $D \in \mathbb{C}^{m^{2} \times 9}$ as follows

$$
D=\left[\begin{array}{llll}
\operatorname{vec}\left(K_{1,1}\right) & \operatorname{vec}\left(K_{2,1}\right) & \cdots & \operatorname{vec}\left(K_{3,3}\right)
\end{array}\right]
$$


where $\operatorname{vec}(\cdot)$ is the matrix to vector operator. Additionally, under suitable assumptions [5,7,9], $D$ can be written out as a sum of contributions of $S$ scattering mechanisms (SMs)

$$
D=\Phi \sum_{s=1}^{S} \theta_{s} C_{s}^{T}=\Phi Z
$$

where $\Phi \in \mathbb{C}^{m^{2} \times n}$ is a partial Fourier matrix, $\theta_{s} \in \mathbb{R}_{\geq 0}^{n}$ accounts for the vertical power distribution of the $s$ th $\mathrm{SM}$ (i.e. its vertical structure), $C_{s} \in \mathbb{C}^{9}$ refers to the polarimetric signature of the $s$ th SM (which can be rearranged into a 3 by 3 polarimetric covariance matrix), and hence $Z \in \mathbb{C}^{n \times 9}$. Accordingly, we will focus on reconstructing $Z$, albeit always driven by the structure conveyed by $\theta_{s}$ and $C_{s}$. Further, even though (2) clearly neglects any source of decorrelation, this aspect will be incorporated as a part of the tomographic reconstruction (see Section 4).

\section{MULTISIGNAL CS}

Multisignal CS enables the joint recovery of signal ensembles by exploiting intersignal structural correlations. It generalizes the concept of a signal being sparse to the concept of an ensemble of signals being jointly sparse [10, 11]. In particular, it proposes taking linear measurements of the form $B=A X+Y$; where $X \in \mathbb{C}^{n \times L}$ indicates $L$ sparse signals of interest that exhibit common support, $A \in \mathbb{C}^{m \times n}$ is a sensing matrix with $m$ usually much smaller than $n$, and $Y \in \mathbb{C}^{m \times L}$ is an unknown perturbation term. The theory asserts that, under suitable conditions $[2,10,12], X$ can be recovered by mixed norm minimization

$$
\min _{\widetilde{X}}\|\widetilde{X}\|_{2,1} \text { subject to }\|A \widetilde{X}-B\|_{F} \leq \varepsilon
$$

where $\varepsilon$ is an upper bound on the perturbation level, $\|\cdot\|_{F}$ is the Frobenius matrix norm, and $\|\cdot\|_{2,1}$ is a mixed norm (sum of the $L_{2}$ norms of the rows of a matrix) that basically promotes sparsity along columns, while minimizing the energy along rows. As a consequence, joint reconstruction guarantees recovery, even when $m<n$, by promoting row sparsity.

\section{COVARIANCE FITTING VIA MULTISIGNAL CS AND POLARIMETRIC SIGNATURE AWARENESS}

In this section, we formulate the reconstruction of $Z$ as defined in (2) from a multisignal CS perspective. To that end, we first observe that forested areas are generally dominated by few effective SMs. Specifically, the assumption of simply two SMs has recently proved to be a valid approximation $[7,9,13]$. In addition, the power distribution $\theta_{s}$ of these different SMs is quite regular [7,8,13,14], thereby giving rise to sparse representations in the wavelet domain and allowing for a CS viewpoint [5]. Consequently, if we let $W \in \mathbb{R}^{n \times n}$ be a sparsifying basis for $\theta_{s}$, with $s \leq 2$, it follows that $Z$ can be represented by a row-sparse matrix $\alpha \in \mathbb{C}^{n \times 9}$, such that $Z=W^{T} \alpha$. Incidentally, the condition $s \leq 2$ can be readily satisfied by replacing $D$ with its best rank- 2 approximation, which will be denoted by $D_{2}$. As thoroughly discussed in [7], this can be efficiently obtained by means of a singular value decomposition (SVD).

\subsection{The Naive Approach}

As a stepping-stone towards a robust formulation, we can simply take equations (2) and (3), and let $B=D_{2}, A=\Phi W^{T}$, $X=\alpha$. Then, we could recover $\alpha$ by carrying out the following optimization

$$
\min _{\widetilde{\alpha}}\|\widetilde{\alpha}\|_{2,1} \text { subject to }\left\|\Phi W^{T} \widetilde{\alpha}-D_{2}\right\|_{F} \leq \varepsilon
$$

where $\varepsilon$ is an upper bound on the model mismatch that potentially captures any source of decorrelation as well as insufficient level of multi-looking [5]. In turn, we would compute $\widetilde{Z}=W^{T} \widetilde{\alpha}$. Although intuitive and simple, this approach falls short of ideal, in that it ignores the inherent properties and low dimensionality of the subspace of polarimetric signatures, which is the subject of Section 4.2.

\subsection{The Data Adaptive Approach}

As previously mentioned, the SVD of $D$ provides an efficient way of computing its best rank-2 approximation. Nonetheless, of equal importance is the fact that the adjoint of the corresponding right singular vectors defines a data adaptive orthonormal basis for the unknown polarimetric signatures [7]. As a result, if we consider only two SMs, i.e. $D_{2}$, it follows that every row of $Z$ will be bound to lie in a two-dimensional subspace. And so, once two orthonormal vectors, $v_{1}$ and $v_{2} \in \mathbb{C}^{9}$, that span the polarimetric space have been obtained and a matrix $V \in \mathbb{C}^{2 \times 9}$

$$
V=\left[\begin{array}{c}
v_{1}^{T} \\
v_{2}^{T}
\end{array}\right]
$$

has been formed, we can formulate a polarimetric signature aware reconstruction as

$$
\min _{\widetilde{\beta}}\|\widetilde{\beta}\|_{2,1} \text { subject to }\left\|\Phi W^{T} \widetilde{\beta} V-D_{2}\right\|_{F} \leq \varepsilon
$$

where $\beta \in \mathbb{R}^{n \times 2}$ and proper constraints must be set on the rows of $Z$, so that every row of $Z$ (when rearranged in a 3 by 3 matrix) results in a positive-semidefinite matrix. Just as in Section 4.1, $\varepsilon$ is an upper bound on the model mismatch. Then, we compute $\widetilde{Z}=W^{T} \widetilde{\beta} V$. For a detailed discussion on how to reduce the size of this kind of problem and ensure positive semidefiniteness, we refer the reader to [13] and the references therein. 
Interestingly, this approach can be understood from a slightly different, yet instructive, viewpoint. Specifically, rather than considering $Z$ to be composed of nine 1-D signals (column vectors), we can think of it as one 2-D signal. As a result, $Z$ can be represented in a 2-D basis, readily formed by computing the outer product of all 1-D wavelet basis vectors and all 1-D polarimetric signature basis vectors, i.e. a Kronecker basis. Thus, just as the former allows for sparse expansions of the vertical backscattered power, the latter provides a sparse expansion of the polarimetric signature. However, since we only consider two SMs, the support of the polarimetric signature transform coefficients is known a priori. Finally, we note that, from this perspective, $\Phi$ is bound to take partitioned measurements, in that only one column is measured at a time. For further details on this kind of distributed sensing setting, we refer the reader to [15].

\section{EXPERIMENTAL RESULTS}

For validation purposes, we used a stack of 10 focused and coregistered SAR images obtained by processing fully polarimetric L-band data. These data were acquired by the E-SAR airborne sensor of DLR during a campaign near Dornstetten, Germany, in 2006. Fig. 1 shows the histogram of the corresponding irregular baseline distribution. The center frequency used was $1.3 \mathrm{GHz}$ and the nominal altitude above ground was about $3200 \mathrm{~m}$. The resolutions were $0.66 \mathrm{~m}$ and $2.07 \mathrm{~m}$ in azimuth and range, respectively [8].

Specifically, we recast (6) in Lagrangian form [3], with $\lambda=2$. The sparsifying basis $W$ was based on the Daubechies Symmlet wavelet with 4 vanishing moments and 3 levels of decomposition. Thus, we reconstructed $Z$ at a fixed range distance of $4816.30 \mathrm{~m}$ for several azimuth-range positions. As a result, we obtained slices in the azimuth and elevation directions of dimensions $300 \mathrm{~m}$ by $40 \mathrm{~m}$, respectively. In order for (2) to hold, we computed the sample covariance matrix by taking a $20 \times 20 \mathrm{~m}^{2}$ estimation window. In this respect, we employed the SKP decomposition [7] using different window sizes in order to find, heuristically, the smallest window that allowed for separation of SMs.

Fig. 2 (a), (b), and (c) present the reconstructed tomographic slices for the $h h, v v$, and $h v$ channels (as taken from $Z$ ), respectively. Additionally, Fig. 2(d) shows the correlations between the $v v$ and $h h$ channels, which clearly manifest more strongly at the ground level.

\section{CONCLUSION}

In this paper, we have extended the existing CS based methodologies by considering the multiple dimensions in which polarimetric covariance matrices, i.e. polarimetric signatures, manifest. Thus, we have been able to perform joint recovery by exploiting the fact that vertical structures as well as polarimetric signatures can be sparsely approximated in appro-

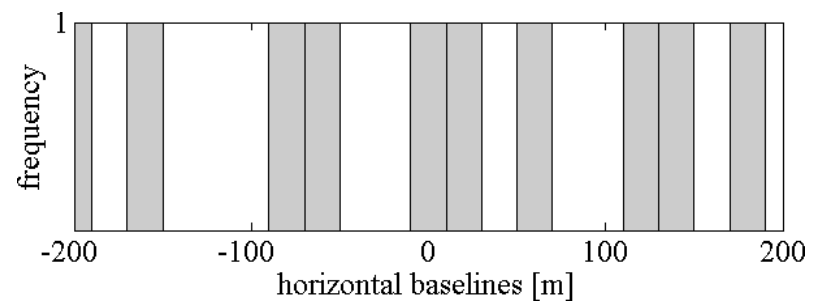

Fig. 1. Histogram of baseline distribution corresponding to 10 irregular parallel passes.

priate domains. For this purpose, we made use of a Wavelet basis as well as a data adaptive basis, respectively. In this regard, we emphasize that the outlined approach is not limited to Wavelets. In fact, any suitable basis could be used. In addition, we have introduced the concept of polarimetric signature awareness, thereby reducing the degrees of freedom to carry out the tomographic reconstruction. Finally, we stress the fact that the implementation of this technique can be effectively tailored by means of existing tools, such as the SKP decomposition.

\section{REFERENCES}

[1] A. Reigber and A. Moreira, "First demonstration of airborne SAR tomography using multibaseline L-band data," IEEE Trans. Geosci. Remote Sens., vol. 38, no. 5, pp. 2142-4152, Sep. 2000.

[2] E. Aguilera, M. Nannini, and A. Reigber, "Multisignal compressed sensing for polarimetric SAR tomography," IEEE Geosci. Remote Sens. Lett., in print, Mar. 2012.

[3] X.X. Zhu and R. Bamler, "Tomographic SAR inversion by L1-norm regularization - The compressive sensing approach," IEEE Trans. Geosci. Remote Sens., vol. 48, pp. 3839-3846, Oct. 2010.

[4] A. Budillon, A. Evangelista, and G. Schirinzi, "Threedimensional SAR focusing from multipass signals using compressive sampling," IEEE Trans. Geosci. Remote Sens., vol. 49, no. 1, pp. 488-499, Jan. 2011.

[5] E. Aguilera, M. Nannini, and A. Reigber, "Wavelet-based compressed sensing for SAR tomography of forested areas," in Proc. EUSAR, 2012.

[6] S. Cloude and E. Pottier, "A review of target decomposition theorems in radar polarimetry," IEEE Trans. Geosci. Remote Sens., vol. 34, no. 2, pp. 498-518, Mar. 1996.

[7] S. Tebaldini, "Algebraic synthesis of forest scenarios from multibaseline PolInSAR data," IEEE Trans. Geosci. Remote Sens., vol. 47, pp. 4132-4142, Dec. 2009.

[8] M. Nannini, R. Scheiber, R. Horn, and A.Moreira, "First 3-D reconstructions of targets hidden beneath foliage by means of polarimetric SAR tomography," IEEE Geosci. Remote Sens. Lett., vol. PP, no. 99, pp. 1-5, Jul. 2011.

[9] S. Tebaldini and F. Rocca, "Multibaseline polarimetric SAR tomography of a boreal forest at P- and L-bands," IEEE Trans. Geosci. Remote Sens., vol. 50, no. 1, pp. 232-246, Jan. 2012.

[10] Y.C. Eldar and H. Rauhut, "Average case analysis of multichannel sparse recovery using convex relaxation," IEEE Trans. Inf. Theory, vol. 56, pp. 505-519, Jan. 2010.

[11] D. Baron, M. Duarte, M. Wakin, S. Sarvotham, and R. Baraniuk, "Distributed compressive sensing," Computing Research Repository, vol. abs/0901.3403, 2009. 


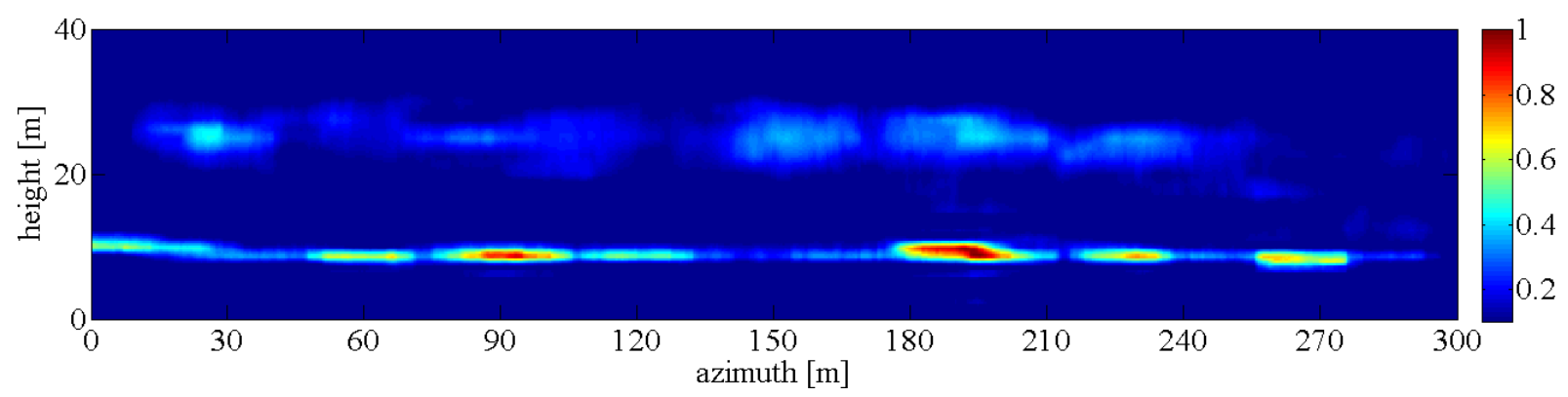

(a)

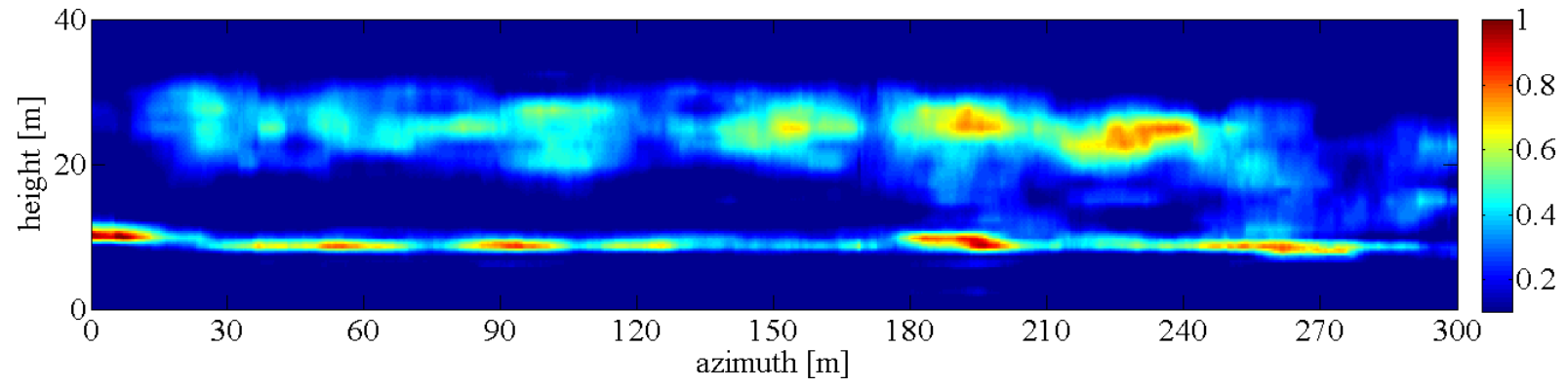

(b)

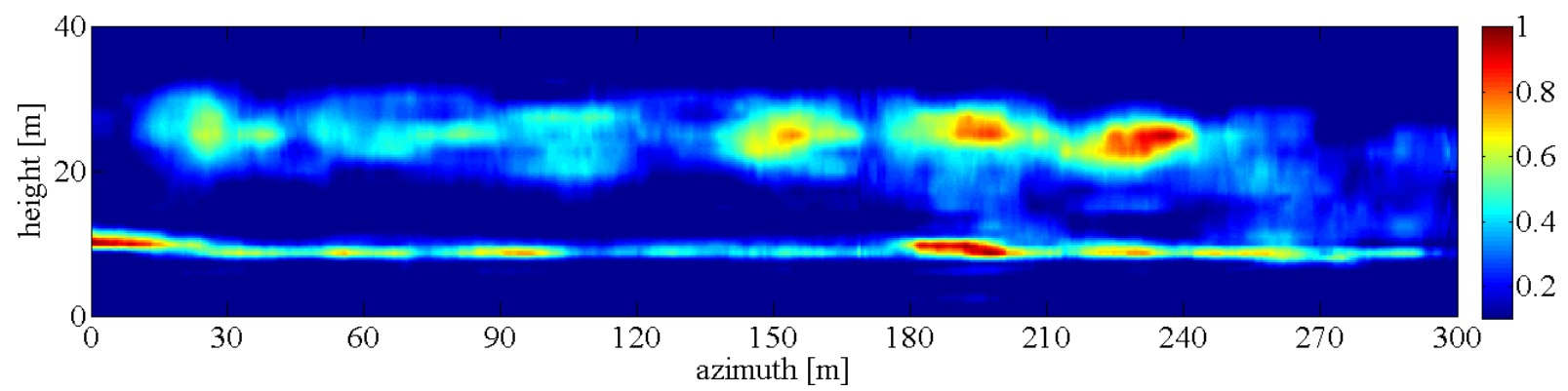

(c)

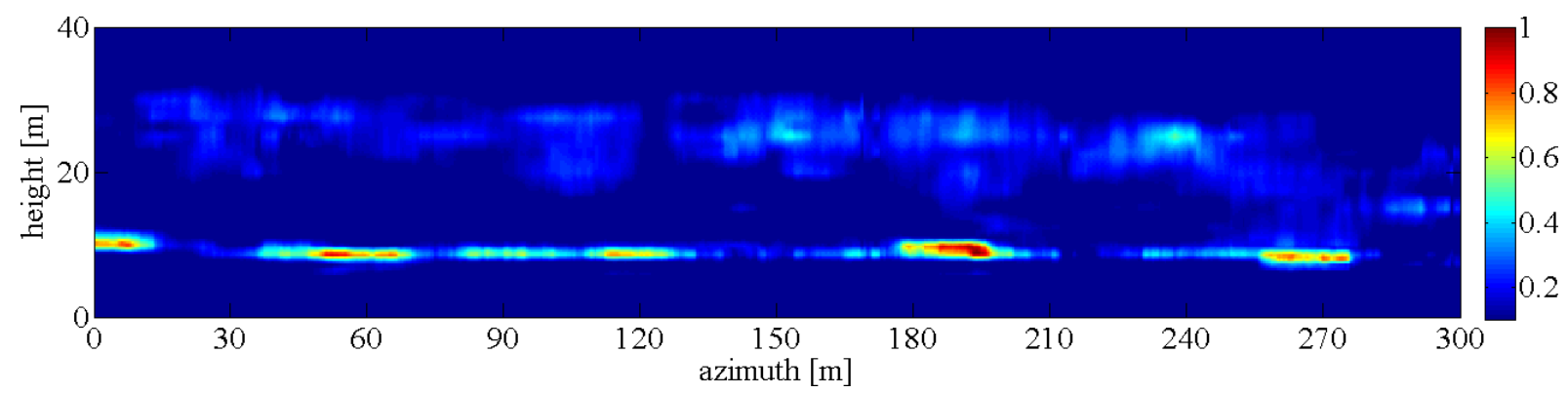

(d)

Fig. 2. Tomographic slices in the Lexicographic basis obtained by means of the data adaptive CS approach (300 $\mathrm{m}$ by $40 \mathrm{~m}$ ) using a $20 \times 20 \mathrm{~m}^{2}$ window (normalized using the maximum value of the tomogram): (a) $h h$ channel, (b) $v v$ channel, (c) $h v$ channel, and (d) correlations between the $v v$ and $h h$ channels.

[12] Y.C. Eldar and M. Mishali, "Robust recovery of signals from a structured union of subspaces," IEEE Trans. Inf. Theory, vol. 55, pp. 5302-5316, 2009.

[13] S. Tebaldini, "Single and multipolarimetric SAR tomography of forested areas: A parametric approach," IEEE Trans. Geosci. Remote Sens., vol. 48, no. 5, pp. 2375-2387, May. 2010.
[14] J. Hagberg, L. Ulander, and J. Askne, "Repeat-pass SAR interferometry over forested terrain," IEEE Trans. Geosci. Remote Sens., vol. 33, no. 2, pp. 331-340, Mar. 1995.

[15] M. Duarte and R. Baraniuk, "Kronecker compressive sensing," IEEE Trans. Image Process., vol. 21, no. 2, pp. 494-504, Feb. 2012. 